\title{
Study on the Key Technology of 3D Printing Pure Titanium Metal
}

\section{Porcelain Teeth}

\author{
Jinjun Tang ${ }^{a}$, Yulei Li ${ }^{b}$,Yue, lai Dai ${ }^{c}$, Xiaoyu Jin ${ }^{d}$, Qun Wang $^{\mathrm{e}}$
}

Ningbo Sub-academy of the National Weapons Science Research Academy, Ningbo 315103,China

ajinjun.tang@163.com, bliyulei7395@163.com, 'daiyuelai_1988@126.com,

dinxiaoyu_nb@163.com, ewangqun_yz@163.com

Key words: laser rapid forming; titanium; metallurgical; 3D printing technology

Abstract: Using laser scanning system for preparation of abutment body of data acquisition and accuracy analysis for the reconstruction of 3D laser scanner abutment preparations and by the method of reverse engineering established the 3D digital model of the simulated abutment, using professional software to simulation of abutment shape completes calculation machine aided design base crown. At the same time, the process parameters of laser rapid forming of pure titanium metal powder were tested by orthogonal design, and the process parameters were optimized by single pass multi-layer cladding forming experiment.

\section{Introduction}

The progress of oral medicine is continuous, to a large extent, with the updating of the oral materials and manufacturing technology[1],2], the great changes have occurred. After a long-term clinical studies, pure titanium material because of its excellent properties of electrochemical corrosion resistance, good biological reaction, suitable mechanical strength, low density and low elastic modulus is currently the best medical dental metal materials, and has been widely used in PFM crowns, denture base, crown and bridge, block body material of Stomatology of preparation[3].

3D printing pure titanium porcelain teeth, to laser gain material manufacturing technology as the foundation is product of the combination of laser technology and NC manufacturing technology, to achieve full density complex metal parts rapid mold manufacturing, compared with the preparation technology of the traditional system[4], the technique has short processing cycle, low manufacturing cost, high flexibility, forming excellent mechanical properties, processing advantages such as the complicated part is at present domestic and foreign manufacturing industry research hot spot, the dental restorations in production field has great application potential[5].

\section{Materials and Methods}

The laser rapid prototyping technology of pure titanium metal substrate is a kind of process parameter, which can achieve the best effect, that is, the influence of process parameters on the forming precision and surface condition. Laser power has a significant influence on the height and width of the single track cladding. With the increase of the laser power, the height and width of the single channel cladding are increased. This experiment to laser power, laser beam scanning speed, powder rate of three factors as the control factors, using the method of orthogonal design with three factors and four levels to arrange the test, as shown in Table 1 shows, the exploration of pure 
titanium powder single pass laser rapid forming parameters.

Tab.1 Control factor in experiment

\begin{tabular}{|c|c|c|c|}
\hline \multirow{2}{*}{ level } & \multicolumn{3}{|c|}{ Factor } \\
\cline { 2 - 4 } & laser power $(\mathrm{W})$ & $\begin{array}{c}\text { scanning speed } \\
(\mathrm{mm} / \mathrm{s})\end{array}$ & $\begin{array}{c}\text { Powder feeding } \\
\text { speed }(\mathrm{g} / \mathrm{min})\end{array}$ \\
\hline 1 & 400 & 7 & 2.5 \\
\hline 2 & 550 & 9 & 3.0 \\
\hline 3 & 700 & 11 & 3.5 \\
\hline 4 & 850 & 13 & 4.0 \\
\hline
\end{tabular}

Laser rapid forming laminated manufacturing technology, layer thickness of the choice relationship to the $\mathrm{Z}$ axis direction of forming precision and multi-layer cladding, each layer cladding substrate preheating temperature and cumulative effect of different, it is necessary to to study the influence on the forming precision of the layer thickness of, research study of multilayer cladding layer thickness selection, and further optimize the processing parameters of the crown base.

\section{Results and discussion}

Laser rapid forming laminated manufacturing technology, layer thickness of the choice relationship to the $\mathrm{Z}$ axis direction of forming precision and multi-layer cladding, each layer cladding substrate preheating temperature and cumulative effect of different, it is necessary to to study the influence on the forming precision of the layer thickness of, research study of multilayer cladding layer thickness selection, and further optimize the processing parameters of the crown base.

This experiment to laser power, laser beam scanning speed, powder rate of three factors as the control factors, using the method of orthogonal design with three factors and four levels to arrange the test, as shown in Figure 1 and Figure 2, explore the pure titanium powder single monolayer of laser rapid forming parameters.

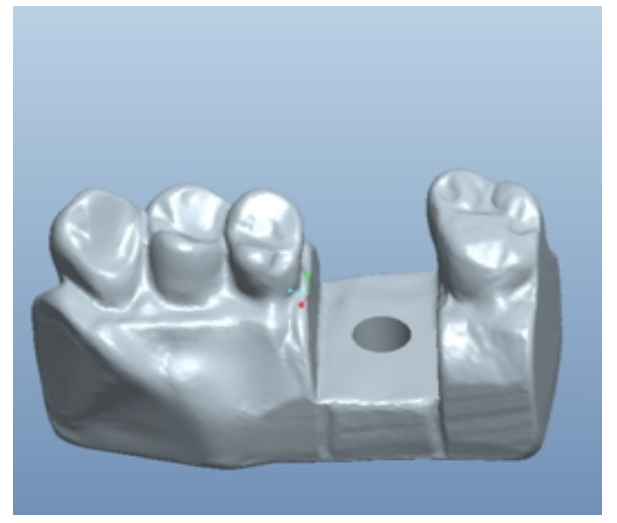

(a)

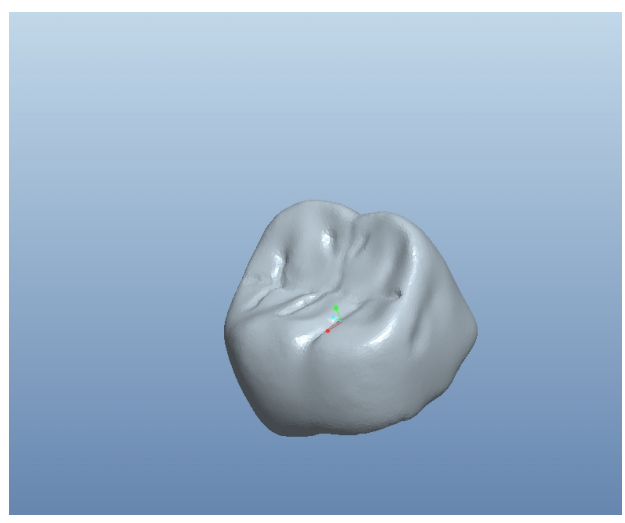

(b)

Fig.1 Digital model of 3D printing porcelain teeth

Laser rapid forming laminated manufacturing technology, layer thickness of the choice relationship 
to the $\mathrm{Z}$ axis direction of forming precision and multi-layer cladding, each layer cladding substrate preheating temperature and cumulative effect of different, it is necessary to to study the influence on the forming precision of the layer thickness of, research study of multilayer cladding layer thickness selection, and further optimize the processing parameters of the crown base.

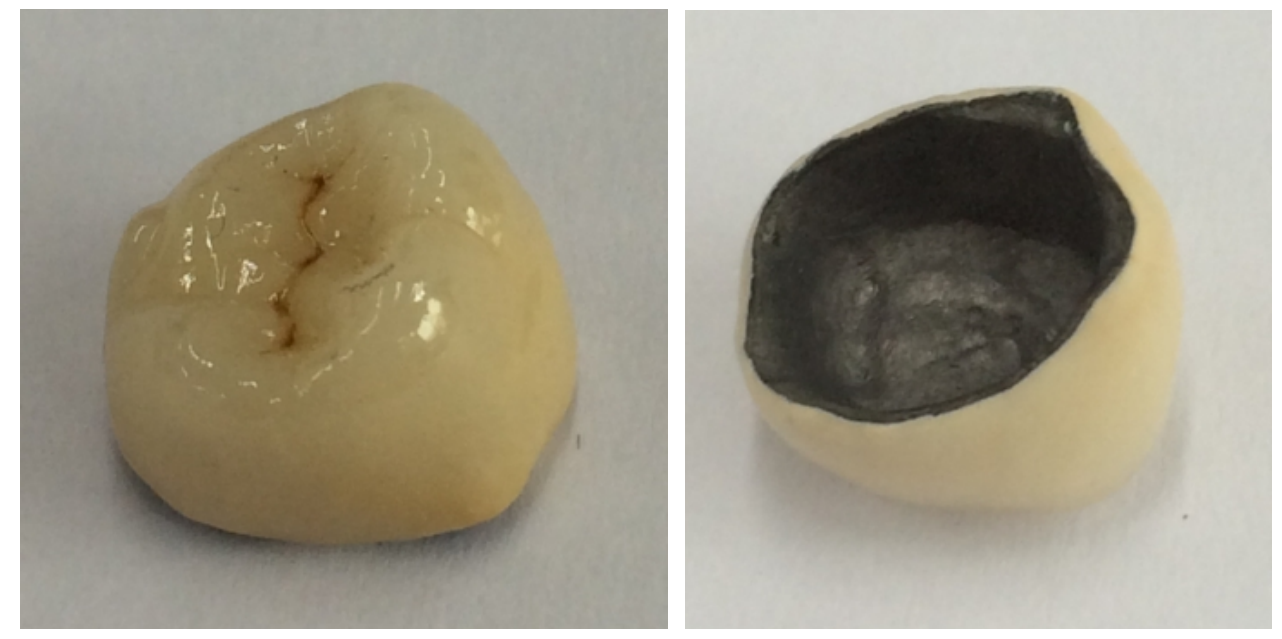

Fig.2 3D print Porcelain teeth sample

When the temperature of the melting point of the porcelain is higher than that of the porcelain, the porcelain is in the molten state, and there is no stress at the interface between the metal and the porcelain. When the temperature is decreased, the stress at the interface is called instantaneous thermal stress due to the difference of the coefficient of thermal expansion. If there is no porcelain crack in the cooling process, the instantaneous thermal stress remains in the system, which is called residual stress, leading to delayed fracture of the porcelain. Porcelain system thermal expansion coefficient difference () can be positive (alpha alpha > gold porcelain) or negative (alpha < alpha gold porcelain). When the delta alpha >, repair body cooling contraction of the metal is greater than porcelain contraction, in porcelain formation pressure stress, because of porcelain with a strong ability to work under pressure, the pressure should not cause porcelain crack, but the ceramometal combine advantageous. Instead, when alpha < Delta, restorations cooling and contraction of the metal is less than the contraction of the porcelain, porcelain formation stress, due to the low tensile strength of the porcelain, the tensile stress is easy to porcelain fracture occurs.

In process of porcelain fused to metal crown base intends to use super porcelain Ti-22 titanium porcelain system, in titanium test piece middle part sequentially coated bonding porcelain $0.2 \mathrm{~mm}$, opaque porcelain $0.2 \mathrm{~mm}$, the dentin porcelain $0.6 \mathrm{~mm}$, and the self-made fixture control porcelain thickness and sintering conditions from tab, casting forming pure titanium cast Ti) as the control group. 


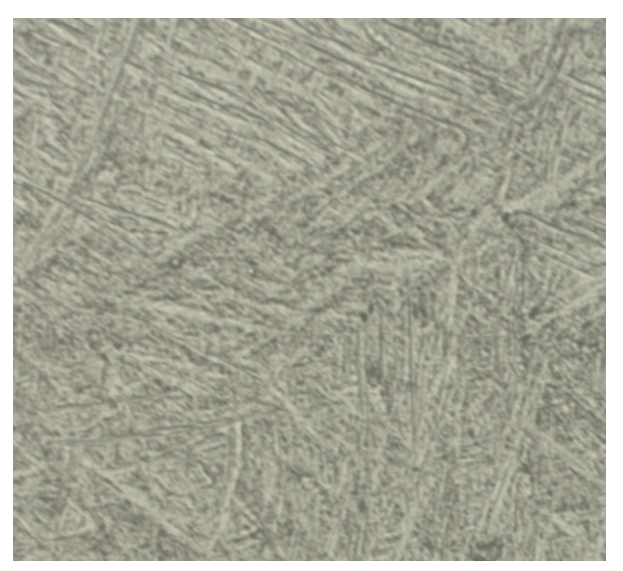

(a) $150 \mathrm{w} ; 2000 \mathrm{~mm} / \mathrm{s} ; 0.1 \mathrm{~mm}$

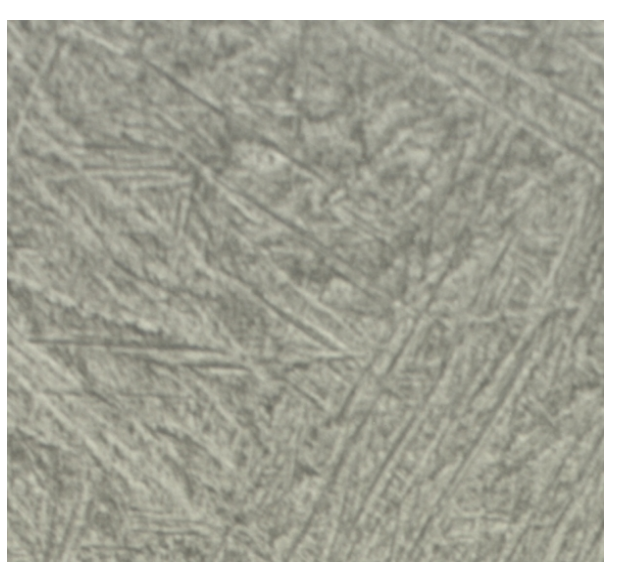

(b) $150 \mathrm{w} ; 2000 \mathrm{~mm} / \mathrm{s} ; 0.2 \mathrm{~mm}$

Fig.3 Microstructure under different circumstances-500X

\section{Conclusion}

Made of different common, $4 \mathrm{~L} / \mathrm{min}-8 \mathrm{~L} / \mathrm{min}, 3 \mathrm{~g} / \mathrm{min} 7 \mathrm{~mm} / \mathrm{s}-9 \mathrm{~mm} / \mathrm{s}$ in order to study the cladding layer thickness on pure titanium metal basal crowns of laser rapid forming quality, the proposed in the laser power of $850 \mathrm{~W}$, scanning speed, powder rate spot diameter $0.8 \mathrm{~mm}$, powder feeding flow process conditions, with $0.05 \mathrm{~mm}, 0.1 \mathrm{~mm}, 0.15 \mathrm{~mm}$ thick layer of laser rapid prototyping, the base crown perpendicular to the scanning direction and ripped up to observe three different parameters monoblock forming quality, self curing plastic embedding, trimmed samples in accordance with the metallographic section by waterproof sandpaper polished to observe the different thickness of cladding cross section morphology, and ultimately determine the appropriate 3D printing pure titanium metal porcelain teeth optimal layer thickness.

\section{References}

[1]Contreras EF, Henriques GE, Giolo SR, Nobilo MA. Fit of cast commercially pure titanium and Ti-6Al-4V alloy crowns before and after marginal refinement by electrical discharge machining[J].J Prosthet Dent, 2002, 88(5): 467-472

[2]Zein I, Hutmacher DW, Tan KC, Teoh SH. Fused deposition modeling of novel scaffold architectures for tissue engineering applications. Biomaterials, 2002, 23(4):1169-85

[3]Santos EC, Osakada K, Shiomi M. Microstructure and mechanical properties of pure titanium models fabricated by selective laser melting[J]. Journal of Mechanical

Engineering Science, Proceedings of ImechE,2004,218C(7):711-719

[4]Peng L, Taiping Y, Sheng L. Direct laser fabrication of nickel alloy samples[J]. Int J Mach Tools Manuf,2005,45(11):1288-94

[5]Hollander DA, Walter MV, Wirtz TT. Structural, mechanical and in vitro characterization of individually structured Ti-6Al-4V produced by direct laser forming[J].Biomaterials,2006, 27:955-963 